\title{
Predictive factors for relapse in adults with dermatomyositis
}

\author{
Hicham Titou ${ }^{1}$, Fatima Zahra Chahboun ${ }^{1}$, Tarik Hanafi ${ }^{1}$, Ahmed Bouhamidi², Youssef Zemmez³, Naoufal Hjira ${ }^{1}$, \\ Mohammed Boui ${ }^{1}$
}

${ }^{1}$ Department of Dermatology, Mohammed V Military Training Hospital, Mohammed V University, Rabat, Morocco. ${ }^{2}$ Department of Dermatology, Avicenna Military Hospital, Marrakesh, Morocco. ${ }^{3}$ Department of Dermatology, Fifth Military Hospital, Guelmim, Morocco.

\begin{abstract}
Introduction: Dermatomyositis (DM) is a severe disease that can be chronic with relapses and severe morbidity. The objective of this study was to analyze clinical features at presentation that predict non-paraneoplastic DM relapse in adult patients.

Methods: A multicenter retrospective study focused on 33 patients diagnosed with DM and treated using corticosteroids from 2000 to 2015 . This study also aimed to compare patients with and without relapse based on some predictive factors such as demographic characteristics and clinical symptoms. A Cox model was used to estimate hazard ratios (HRs).

Results: We identified 33 patients with an average age of $41.8 \pm 12$ years (range 18-68 years). The sex ratio of females to males was $2: 1$. During the 13.5 -month follow-up period, two (6\%) patients died and $21(63.6 \%)$ suffered from a relapse. In univariate analysis, skin ulceration in the initial presentation of disease $(H R=3.1$, range 1.1 to 15.1$)$ was significantly associated with a relapsing course.
\end{abstract}

Conclusions: This study demonstrates that in the initial presentation of the disease skin ulceration is significant in allowing prediction of the occurrence of DM relapse.

Keywords: dermatomyositis, ulceration, relapse, Morocco

Received: 23 December 2019| Returned for modification: 4 February 2020 | Accepted: 10 February 2020

\section{Introduction}

Dermatomyositis (DM) belongs to a group of diseases known as idiopathic inflammatory myopathies (1). It causes inflammatory damage to both the skin and the striated muscles, leading to muscle weakness, increasing muscle enzymes, and showing myogenic signs on electromyogram (EMG).

In 1975, Bohan and Peter proposed five major diagnostic criteria to define $\mathrm{DM}(2,3)$. The clinical symptoms of patients receiving treatment are temporarily improved, but the majority experience a relapse and/or chronic progression $(4,5)$. Recently, some clinical forms with classic skin lesions of DM have been described with minimal (hypomyopathic) or no muscle weakness (amyopathic), termed clinically amyopathic DM (CADM) (6). However, the connection between CADM and the occurrence of relapse has not yet been determined $(7,8)$. DM relapse remains difficult to characterize because of a lack of consensus on its definition $(4,9)$. In a practical sense, a relapse occurs when certain symptoms recur or worsen after clinical improvement under initial treatment.

What makes DM a severe disease is its spontaneous progression, eventual association with cancer, and treatment-induced complications $(10,11)$.

Prognostic factors reported for patients suffering from polymyositis/dermatomyositis (PM/DM) include old age, a long duration between the occurrence of the first symptoms and the start of treatment, male sex, CADM, dysphagia, interstitial lung disease, heart disease, and malignancy (12-14). Moreover, these prognostic factors make it possible to predict the occurrence of a relapse and other outcomes such as death, resistance to treatment, and disease progression. These prognostic factors have been identified thanks to some medical studies that were carried out on heterogeneous populations, including patients diagnosed with para- neoplastic PM or DM.

This study analyzes the long-term outcomes of patients diagnosed with non-paraneoplastic DM and considers possible factors responsible for relapses.

\section{Patients and methods}

We conducted a multicenter retrospective cohort study of patients diagnosed with DM. This study was conducted at three dermatology departments, including Mohamed V Military Training Hospital in Rabat, Avicenna Military Hospital in Marrakesh, and Fifth Military Hospital in Guelmim from January 1st, 2000 to January 1st, 2015. In this analysis, the clinical inclusion criteria were a diagnosis of DM per Bohan and Peter $(2,3)$, systemic corticosteroid treatment, and age $>18$ years at diagnosis.

During this study, we excluded patients diagnosed with paraneoplastic DM, which is considered a cancer diagnosis in the 2 years following the diagnosis of DM (15), because the evolution of this form of DM is related to the effectiveness of cancer therapy. We also excluded patients not treated with corticosteroids, and those with overlap syndrome and juvenile DM (DMJ).

Hence, the following information was collected by one investigator: medical antecedents, sociodemographic characteristics (age and sex), clinical data (clinical presentation and treatment received), medical follow-up (relapse, time between diagnosis and relapse, complications, and death), and paraclinical data: antinuclear antibodies, level of creatine kinase (CK), thoracic tomodensitometry (TDM), and EMG.

We evaluated the gravity of cutaneous lesions with the help of the Cutaneous Disease Area Severity Index (CDASI) (16). The CDASI value can range from o to 132. Moreover, it depends on lesion localization, the intensity of the cutaneous eruption, the presence 
of periungual manifestations, and Gottron's papules.

All muscle biopsies were addressed to the anatomical pathology department of Mohamed V Military Training Hospital in Rabat. To evaluate the severity of the histological lesions, we retrospectively relied on a validated scoring tool developed by the International JDM Biopsy Consensus Group $(17,18)$. Basic changes in each domain of this tool were scored: inflammatory, vascular, muscle fiber, and connective tissue. A visual analogue scale (VAS) was used to evaluate individual symptoms' severity.

Patients were divided into two groups based on whether a DM relapse had occurred or not. A DM relapse was defined as a reoccurrence of muscular or skin manifestation after an improvement of the patients, followed by a change in treatment: an increase of at least 50\% in the dosage of corticosteroids, adding an immunosuppressant, or switching to another immunosuppressant.

\section{Statistical analysis}

To perform the statistical analysis, we used the Statistical Package for the Social Sciences (SPSS) version 20.0. Student's $t$-test was used to compare the continuous variables, and the chi-squared test was used to compare the percentage of two independent groups. The quantitative variables with asymmetrical distributions were analyzed using the Mann-Whitney $U$ test to compare two independent groups. In addition, the Kaplan-Meier method was used to evaluate the probability of survival according to the time in remission. To compare the survival curves in accordance with sex, the logrank test was used. A value of $p$ lower than 0.05 was regarded as significant.

\section{Results}

Altogether, 48 patients were identified with DM. Among them, nine $(18.7 \%)$ were suffering from a paraneoplastic DM. However, among the 39 patients diagnosed with DM, only 33 (84.6\%) met our inclusion criteria.

According to this study, the mean age of onset of symptoms was $41.8 \pm 12$ years (range 18-68 years), with a female predominance (66.7\%; Table 1). The difference in mean age between sexes was not statistically significant $(p=0.6)$. The medical follow-up duration was $13.5 \pm 22.3$ months (range 1 month 9 years to 3 months). The patients were seen between 2 weeks and 12 months (median 3, range 1-4 months) after the beginning of the symptoms, and 28 $(84.8 \%)$ of the patients were seen during the first 6 months. The DM diagnosis was definite in $29(87.8 \%)$ patients, probable in two $(6.1 \%)$ patients, and possible in two $(6.1 \%)$. Table 1 presents the principal clinical and paraclinical characteristics of patients.

Severe muscle involvement involved dysphagia $(n=12,36.4 \%)$, swallowing disorders $(n=3,9.1 \%)$, and dysphonia $(n=7,21.2 \%)$. No patient had interstitial lung disease or cardiac involvement.

Antinuclear antibodies were positive $(>1 / 80)$ for $18(60.0 \%)$ patients. However, anti-SSA antibodies were positive for only three $(10.7 \%)$ patients among the 28 that had already benefitted from the test. Absence of data related to anti-JO1 and anti-Mi2 for all patients precluded an evaluation of these antibodies by univariate analysis (Table 2).

Prednisone at a dosage of 1 to $2 \mathrm{mg} / \mathrm{kg} /$ day was the initial treatment for all patients. Six (18.2\%) patients received bolus therapy with methylprednisolone (250-500 mg/day) during the first hos-

Table 1 | Initial characteristics of the study population $(n=33)$.

\begin{tabular}{|c|c|c|c|}
\hline Initial characteristics & Total $n(\%)$ & Relapse $n(\%)$ & No relapse $n(\%)$ \\
\hline \multicolumn{4}{|l|}{ Sex } \\
\hline Male & $11(33.3)$ & $7(33.3)$ & $4(33.3)$ \\
\hline Female & $22(66.7)$ & $14(66.7)$ & $8(66.7)$ \\
\hline Age, years $($ mean $\pm S D)$ & $41.8 \pm 12$ & $43.6 \pm 11$ & $38.7 \pm 13.4$ \\
\hline Time to diagnostic, months, median (range) & $3(1-4)$ & $2(1-4)$ & $3(1-6.25)$ \\
\hline \multicolumn{4}{|l|}{ Bohan and Peter criteria } \\
\hline Skin rash & $33(100.0)$ & $21(100.0)$ & $12(100.0)$ \\
\hline Proximal and symmetrical weakness & $28(84.8)$ & $19(90.5)$ & $9(75.0)$ \\
\hline CK elevation & $24(72.7)$ & $18(85.7)$ & $6(50.0)$ \\
\hline Electromyogram abnormalities & $25(75.8)$ & $16(76.2)$ & $9(75.0)$ \\
\hline Specific muscle biopsy & $21(63.6)$ & $14(66.7)$ & $7(58.3)$ \\
\hline \multicolumn{4}{|l|}{ Comorbidities } \\
\hline Hypertension & $2(6.0)$ & $2(9.5)$ & $0(0)$ \\
\hline Obesity & $3(9.1)$ & $2(9.5)$ & $1(8.3)$ \\
\hline Smoking & $5(15.1)$ & $2(9.5)$ & $3(25.0)$ \\
\hline \multicolumn{4}{|l|}{ Dermatological abnormalities } \\
\hline Gottron's papules & $15(45.4)$ & $8(38.1)$ & $7(58.3)$ \\
\hline Periungual involvement & $6(18.1)$ & $4(19.0)$ & $2(16.7)$ \\
\hline Ulceration & $8(24.2)$ & $6(28.6)$ & $2(16.7)$ \\
\hline CDASI $>20$ & $14(42.4)$ & $8(38.1)$ & $6(60.0)$ \\
\hline \multicolumn{4}{|l|}{ Severity of muscle involvement } \\
\hline Dysphagia & $12(36.3)$ & $9(42.9)$ & $3(25.0)$ \\
\hline Swallowing disorders & $4(12.1)$ & $2(9.5)$ & $2(16.7)$ \\
\hline Dysphonia & $7(21.2)$ & $5(23.8)$ & $2(16.7)$ \\
\hline Soft palate paralysis & 0 & 0 & 0 \\
\hline \multicolumn{4}{|l|}{ Other manifestations } \\
\hline Arthralgia & $11(33.3)$ & $6(28.6)$ & $5(41.7)$ \\
\hline Dyspnea & $3(9.1)$ & $3(14.3)$ & 0 \\
\hline Alopecia & $3(9.1)$ & $1(4.8)$ & $2(16.7)$ \\
\hline \multicolumn{4}{|l|}{ Findings from initial investigations } \\
\hline CK elevation & $20(60.6)$ & $14(66.7)$ & $6(50.0)$ \\
\hline CK level > 5,000 IU & $3(9.1)$ & $1(4.8)$ & $2(16.7)$ \\
\hline Interstitial syndrome & 0 & 0 & 0 \\
\hline Electrocardiogram abnormalities & 0 & 0 & 0 \\
\hline
\end{tabular}

$\mathrm{SD}=$ standard deviation, $\mathrm{CK}=$ creatine kinase, $\mathrm{CDASI}=$ Cutaneous Disease Area Severity Index. 
pitalization because of dysphagia, swallowing disorder, or dysphonia. Eight (24.2\%) patients received methotrexate weekly. Finally, $11(33.3 \%)$ patients received hydroxychloroquine $(400 \mathrm{mg} /$ day) in association with corticosteroids.

\section{Histological score}

Muscle biopsies were performed on 23 patients. However, only 14 results of muscle biopsies were available for analysis (Table 3). The International JDM Biopsy Consensus Group score showed severe typical DM features in all 14 patients. Analysis of the inflammatory domain showed a typical inflammatory infiltrate with a median score of 5.5 (range 0-12). These infiltrates were localized between the perimysium and endomysium. The analysis of the muscular fiber domain included perifascicular and non-perifascicular atrophy and regeneration/degeneration/necrosis, which scored 6 (range 0-8). Concerning the vascular domain, 7/14 patients showed ischemia with infarcts, $11 / 14$ showed microvascular involvement, and 2/14 showed capillary drop. No patient had arterial abnormalities. The median VAS score for severity of individual symptoms was 6 (score $0-10$ ).

\section{Relapses and survival}

Two-thirds (63.6\%) of patients experienced at least one relapse, and the mean duration to the first relapse was $12 \pm 14.4$ months (range 2 months to 4 years). The number of relapses per patient varied between 1 and 6 . At relapse onset, the mean dosage of corticosteroid was $0.14 \mathrm{mg} / \mathrm{kg} /$ day \pm 0.3 . For one (3.2\%) patient, a relapse was documented after 5 years of survival in remission without any treatment. Treatments given to patients for the first relapse were methotrexate $(n=8)$, azathioprine $(n=2)$, corticosteroid in monotherapy $(n=5)$, and corticosteroid associated with methotrexate $(n=6)$. In univariate analysis, the presence of skin ulceration (HR 3.1, range 1.1-15.1) was significantly associated with relapse (Table 2). Due to the small sample size, multivariate analysis was not performed. The mean duration of survival in remission among females was longer than that of males (15.5 months vs. 14.4 months; $p=0.7$; Fig. 1); however, this difference was not statistically significant.

\section{Complications}

One (3\%) patient died due to an ischemic cerebrovascular acci-

Table 2 | Factors showing a significant association or a trend toward an association with relapse by univariate analysis $(n=33)$.

\begin{tabular}{lccc}
\hline Initial characteristics & $\mathrm{HR}$ & $95 \% \mathrm{Cl}$ & $p$-value \\
\hline Ulceration & 3.1 & $1.1-15.1$ & 0.03 \\
Dysphonia & 0.7 & $0.1-13.0$ & 0.80 \\
CDASI $\geq 20$ & 4.1 & $0.6-15.9$ & 0.10 \\
Dyspnea & 3.1 & $0.2-14.2$ & 0.20 \\
Alopecia & 0.7 & $0.1-8.4$ & 0.70 \\
\hline
\end{tabular}

CDASI = Cutaneous Disease Area Severity Index, $\mathrm{HR}=$ hazard ratio, $\mathrm{CI}=$ confidence interval. dent, and one $(3 \%)$ patient died due to severe sepsis. The reasons behind their deaths were not directly related to DM. At least one complication occurred in $20(60.6 \%)$ patients. More than one-third $13(39.3 \%)$ of patients experienced infection: pulmonary tuberculosis $(n=5)$, bacterial pneumonia $(n=5)$, soft tissue abscesses $(n$ $=3)$, cerebral abscess $(n=1)$, and arthritis $(n=2)$. Osseous complications were documented in four (12.1\%) patients: three patients were diagnosed with osteoporosis and one patient with osteopenia. During the period of medical follow-up, four (12.1\%) patients were diagnosed with diabetes and two $(6 \%)$ patients suffered from arterial hypertension.

\section{Discussion}

Our study is based on a 14-year follow-up of a cohort of $33 \mathrm{DM}$ patients treated with systemic corticosteroids in Morocco. In this study, the diagnosis of DM was definite in $87.8 \%$ of our patients.

The distribution of the clinical forms of DM (classic, paraneoplastic, and amyopathic/hypomyopathic) was similar to those of previous studies $(15,19,20)$. This distribution indicates that the selection bias was probably minimal. In this study, patients diagnosed with myopathic or hypomyopathic DM were excluded because they were not treated with corticosteroids at the time of diagnosis of the disease. The patients diagnosed with paraneoplastic DM were also eliminated because anti-cancer treatment may govern the development of this disease. The objective of this work was to analyze the long-term outcomes of patients diagnosed with a non-paraneoplastic DM bearing in mind the possible factors that are responsible for relapses.

In this study, the rate of female patients was high (66.7\%). This result is similar to that found by the majority of previous studies (19-21). In the data presented in this study, the highest DM incidence was observed in the fourth decade of life. This was found by

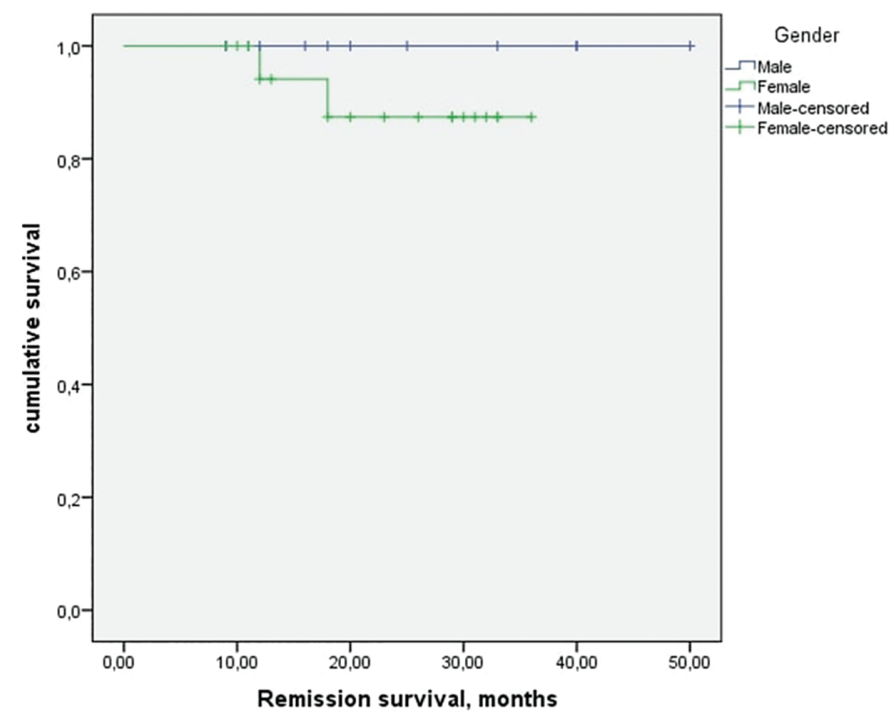

Figure 1 | Remission survival in male and female patients using Kaplan-Meier and logrank comparisons. Data are censored for patient deaths during the study period.

Table $3 \mid$. Muscle biopsy characteristics $(n=14)$ and their association with relapse.

\begin{tabular}{|c|c|c|c|c|c|}
\hline Domain (extreme range) & Median (range) & Relapse $(n=9)$, median (range) & No relapse $(n=5)$, median (range) & $\mathrm{HR}(95 \% \mathrm{Cl})$ & $p$-value \\
\hline Inflammatory domain (0-2) & $7(5-8.25)$ & $7(5.5-10)$ & $6(4.5-7.5)$ & $1.2(0.4-2.9)$ & 0.60 \\
\hline Vascular domain (0-3) & $1(1-2)$ & $1(1-1.5)$ & $1(1-2)$ & $0.9(0.1-25)$ & 0.90 \\
\hline Muscle fiber domain (0-10) & $8(5.7-9)$ & $8(5.5-9)$ & $8(7-9.5)$ & $0.5(0.1-2)$ & 0.30 \\
\hline Connective tissue domain $(0-2)$ & $0(-)$ & $0(-)$ & $0(0-0.5)$ & $0(-)$ & 1 \\
\hline Visual analogue scale $(0-10)$ & $6(4-9)$ & $8(4.5-9)$ & $6(2.5-7.5)$ & $1.2(0.8-1.9)$ & 0.50 \\
\hline Total (0-37) & $22(20-25.2)$ & $22(21-25.5)$ & $21(15.5-25.5)$ & $1.1(0.8-1.6)$ & 0.30 \\
\hline
\end{tabular}

$\mathrm{HR}=$ hazard ratio, $\mathrm{Cl}=$ confidence interval. 
other studies, which reported a similar distribution in the fourth and fifth decades (19-21). Moreover, almost two-thirds (63.6\%) of patients have experienced one relapse. In this regard, other previous studies have reported a rate of relapse between 38 and 65\% (9, 19, 20). Time from DM diagnosis to the first relapse was $12 \pm 14.4$ months, which is in accordance with previous observations (19, 22). In our study, the majority of relapses occurred during the corticosteroid taper. Therefore, close monitoring is necessary during this period. Due to the high rate of relapses observed, we recommend the prolongation of corticosteroid therapy with very slow regression.

We have also shown that skin ulceration at the beginning of the disease is significantly associated with relapse. The majority of these skin ulcerations were located in the buttocks and thighs. Indeed, the ulcers probably reflect an important cutaneous vasculopathy, caused by hypoxia and ischemia of the affected tissues $(23,24)$. Some authors believe that the presence of skin ulcerations is a marker of poor long-term prognosis (25). More particularly, ulcerative or necrotic lesions are more frequent when the DM is associated with cancer $(16,26)$.

The initial result of muscular biopsies was evaluated using a validated scoring tool to reduce bias due to the retrospective nature of this study. Interestingly, these results were not associated with a relapse of DM and were comparable to those of another study conducted in Paris by Vuong et al. (19). Conversely, in his work on 72 muscle biopsies from patients with DMJ, Miles et al. discovered that extensive myopathic changes and central nuclei without basophilia were predictive factors of chronic disease (27).

Other predictive factors of PM/DM relapses have been reported, such as old age, delay in appropriate therapy, pulmonary involvement, dysphonia, skin lesions at the onset of DM, and the presence of positive anti-SS-A autoantibodies (19, 20, 28). In our study, these factors were not statistically associated with DM relapse. Indeed, these risk factors were identified in a heterogeneous group of diseases affecting paraneoplastic DM, polymyositis, DMJ and overlap syndrome. Thus, the results of these studies cannot be compared to our study.

\section{References}

1. Callen JP. Dermatomyositis. Lancet. 2000;355:53-7.

2. Bohan A, Peter JB. Polymyositis and dermatomyositis (first of two parts). N Engl J Med. 1975;292:344-7.

3. Bohan A, Peter JB. Polymyositis and dermatomyositis (second of two parts). N Engl J Med. 1975;292:403-7.

4. Marie I, Hachulla E, Hatron PY, Hellot MF, Levesque H, Devulder B, et al. Polymyositis and dermatomyositis: short-term and long-term outcome, and predictive factors of prognosis. J Rheumatol. 2001;28:2230-7.

5. Taborda AL, Azevedo P, Isenberg DA. Retrospective analysis of the outcome of patients with idiopathic inflammatory myopathy: a long-term follow-up study. Clin Exp Rheumatol. 2014;32:188-93.

6. Sontheimer RD. Would a new name hasten the acceptance of amyopathic dermatomyositis (dermatomyositis siné myositis) as a distinctive subset within the idiopathic inflammatory dermatomyopathies spectrum of clinical illness? J Am Acad Dermatol. 2002;46:626-36.

7. Yamasaki Y, Yamada H, Ohkubo M, Yamasaki M, Azuma K, Ogawa H, et al. Longterm survival and associated risk factors in patients with adult-onset idiopathic inflammatory myopathies and amyopathic dermatomyositis: experience in a single institute in Japan. J Rheumatol. 2011;38:1636-43.

8. Fujisawa T, Hozumi H, Kono M, Enomoto N, Hashimoto D, Nakamura $\mathrm{Y}$, et al. Prognostic factors for myositis-associated interstitial lung disease. PLoS One. 2014;9:e98824.

9. Ponyi A, Constantin T, Balogh Z, Szalai Z, Borgulya G, Molnăr K, et al. Disease course, frequency of relapses and survival of 73 patients with juvenile or adult dermatomyositis. Clin Exp Rheumatol. 2005;23:50-6.
The median survival was comparable between men and women (15.5 months vs. 14.4 months; $p=0.7$ ). However, this difference is not statistically significant. In their study, Watanabe et al. showed that the negative status of autoantibodies specific to myositis (MSA) and the absence of severe muscular weakness necessitating assistance at diagnosis were predictive factors for long survival in remission in patients with PM/DM (21).

During the follow-up period, two patients died. Severe sepsis was the cause of death for the first patient, and an ischemic cerebrovascular accident for the other patient. Many risk factors are reported to be associated with mortality in patients with $\mathrm{PM} / \mathrm{DM}$, including malignant tumors, male sex, old age, and pulmonary diseases (including interstitial lung disease) $(4,5,7)$.

In this study, infections (13/20) were the most common complications. Indeed, several reports have investigated risk factors for infections in patients with connective tissue disease, such as old age, male sex, and pulmonary diseases $(29,30)$. According to a study by Murray et al., infection is the principal cause of mortality in patients with $\mathrm{PM} / \mathrm{DM}$ (31).

Several limitations warrant attention in this work. First, the retrospective design can be responsible for the selection bias of patients. Second, the fact that the disease is rare limited the size of the sample, which allows only a univariate analysis with possible influence of confounding factors. Third, the heterogeneity of populations in most previous studies makes it difficult to compare the risk factor data for relapse.

\section{Conclusions}

Our study evaluated risk factors associated with relapse in a uniform group of adult patients with classic DM. In univariate analysis, the presence of skin ulcerations at presentation of disease significantly predicted a relapsing course. However, a large multicenter cohort study is necessary to assess predictors of classic DM relapse in adults. The results of these studies would be a rationale for adjusting corticosteroid dosage in patients according to the importance of relapse risk.
10. Bohan A, Peter JB, Bowman RL, Pearson CM. Computer assisted analysis of 153 patients with polymyositis and dermatomyositis. Medicine. 1977;56:255-86.

11. Jorizzo 3 rd LJ, Jorizzo JL. The treatment and prognosis of dermatomyositis: an updated review. J Am Acad Dermatol. 2008;59:99-112.

12. Hamaguchi Y, Kuwana M, Hoshino K, Hasegawa M, Kaji K, Matsushita T, et al. Clinical correlations with dermatomyositis-specific autoantibodies in adult Japanese patients with dermatomyositis. Arch Dermatol. 2011;147:391-8.

13. Marie I, Hatron PY, Dominique S, Cherin P, Mouthon L, Menard JF. Short-term and long-term outcomes of interstitial lung disease in polymyositis and dermatomyositis: a series of 107 patients. Arthritis Rheum. 2011;63:3439-47.

14. Bronner IM, van der Meulen MFG, de Visser M, Kalmijn S, van Venrooij WJ, Voskuyl AE, et al. Long-term outcome in polymyositis and dermatomyositis. Ann Rheum Dis. 2006;65:1456-61.

15. Fardet L, Dupuy A, Gain M, Kettaneh A, Chérin P, Bachelez H, et al. Factors associated with underlying malignancy in a retrospective cohort of 121 patients with dermatomyositis. Medicine (Baltimore). 2009;88:91-7.

16. Yassaee M, Fiorentino D, Okawa J, Taylor L, Coley C, Troxel AB, et al. Modification of the cutaneous dermatomyositis disease area and severity index, an outcome instrument. Br J Dermatol 2010;162:669-73.

17. Wedderburn LR, Varsani H, Li CK, Newton KR, Amato AA, Banwell B, et al. International consensus on a proposed score system for muscle biopsy evaluation in patients with juvenile dermatomyositis: a tool for potential use in clinical trials. Arthritis Rheum. 2007;57:1192-201. 
18. Varsani H, Charman SC, Li CK, Marie SK, Amato AA, Banwell B, et al. Validation of a score tool for measurement of histological severity in juvenile dermatomyositis and association with clinical severity of disease. Ann Rheum Dis. 2015; 74:204-10.

19. Vuong V, Duong TA, Aouizerate J, Authier FJ, Ingen-Housz-Oro S, Valeyrie-Allanore L, et al. Dermatomyositis: factors predicting relapse. J Eur Acad Dermatol Venereol. 2016;30:813-8.

20. Tatebe N, Sada KE, Asano Y, Zeggar S, Hiramatsu S, Miyawaki Y, et al. Anti-SS-A/ Ro antibody positivity as a risk factor for relapse in patients with polymyositis/ dermatomyositis. Mod Rheumatol. 2018; 28:141-6.

21. Watanabe E, Gono T, Kuwana M, Terai C. Predictive factors for sustained remis sion with stratification by myositis-specific autoantibodies in adult polymyositis/dermatomyositis. Rheumatology (Oxford). 2019; [Epub ahead of print].

22. Constantin T, Ponyi A, Orban I, Molnar K, Derfalvi B, Dicso F, et al. National registry of patients with juvenile idiopathic inflammatory myopathies in Hungaryclinical characteristics and disease course of 44 patients with juvenile dermatomyositis. Autoimmunity. 2006;39:223-32.

23. Mamyrova G, Kleiner DE, James-Newton L, Shaham B, Miller FW, Rider LG. Lateonset gastrointestinal pain in juvenile dermatomyositis as a manifestation of ischemic ulceration from chronic endarteropathy. Arthr Rheum. 2007;57:881-4.

24. Pachman LM, Cooke N. Juvenile dermatomyositis: a clinical and immunologic study. J Pediatr. 1980;96:226-34.
25. Bowyer SL, Blane CE, Sullivan DB, Cassidy JT. Childhood dermatomyositis: factors predicting functional outcome and development of dystrophic calcification. J Pediatr. 1983;103:882-8.

26. Basset-Seguin N, Roujeau JC, Gherardi R, Guillaume JC, Revuz J, Touraine R. Prognostic factors and predictive signs of malignancy in adult dermatomyositis. A study of 32 cases. Arch Dermatol. 1990;126:633-7.

27. Miles L, Bove KE, Lovell D, Wargula JC, Bukulmez H, Shao M, et al. Predictability of the clinical course of juvenile dermatomyositis based on initial muscle biopsy: a retrospective study of 72 patients. Arthritis Rheum. 2007;57:1183-91.

28. Agarwal SK, Monach PA, Docken WP, Coblyn JS. Characterization of relapses in adult idiopathic inflammatory myopathies. Clin Rheumatol. 2006;25:476-81.

29. Yamazaki H, Sakai R, Koike R, Miyazaki Y, Tanaka M, Nanki T, et al. Assessment of risks of pulmonary infection during 12 months following immunosuppressive treatment for active connective tissue diseases: a large-scale prospective cohort study. J Rheumatol. 2015;42:614-22.

30. Feldman CH, Hiraki LT, Winkelmayer WC, Marty FM, Franklin JM, Kim SC, et al. Serious infections among adult Medicaid beneficiaries with systemic lupus erythematosus and lupus nephritis. Arthritis Rheumatol. 2015;67:1577-85.

31. Murray SG, Schmajuk G, Trupin L, Lawson E, Cascino M, Barton J, et al. A population-based study of infection-related hospital mortality in patients with dermatomyositis/polymyositis. Arthritis Care Res (Hoboken). 2015;67:673-80. 